\title{
Assessment of Correlation Between HLA-B27 Associated Ankylosing Spondylitis With Acute Anterior Uveitis
}

\author{
Sachin Sharma, C.V. Kulkarni, Ashok Yadav, Sweta Tripathi* and Gaurav Shelgaonkar \\ Department of pathology, Mahatma Gandhi Memorial Medical college Indore (M.P) INDIA
}

\section{ABSTRACT}

Background: Human leukocyte antigen HLA -B27 is a class I surface antigen which is encoded by the B locus on the chromosome number 6 in the major histocompatibility complex (MHC) . HLA -B27 antigen is associated with various seronegative arthropathies comprising of Ankylosing spondylitis, reactive arthritis, psoriatic arthritis and inflammatory bowel disease along with acute anterior uveitis. Main aim of our study is to analyze thecorrelation between the HLA -B27 associated Ankylosingspondylitis and uveitis.

Method: Hundredpatients with clinical symptoms and signs of Ankylosing spondylitis and Acute Anterior Uveitis were selected for the study and the blood samples were studied for flowcytometric analysis of HLA-B27/7.

Result: Study suggests thatpatient of AS and positive HLA-B27 antigen have higher incidence of Acute anterior uveitis as compared to patients with AS and negative status of HLA- B27 antigen.

Conclusion: Study suggests that HLA-B27 antigen is associated with both the clinical conditions, Ankylosing spondylitis and uveitis and these two clinical condition are closely related to each other.

Keywords: Ankylosing Spondylitis, Acute Anterior Uveitis , HLA, Major Histocompatibility Complex

\section{Introduction}

The human leukocyte antigen (HLA) system describes the major histocompatibility complex (MHC) in humans. This super locus contains a large number of genes related to immune system functions in humans. The association between the HLA-B27, Ankylosing Spondylitis and

AAU was first described in 1973. ${ }^{[1,2]}$ Among the all known association between HLA -B27 and diseases, the strongest association is seen in Ankylosing spondylitis. The B-27 antigen is present in over $90 \%$ of patients with AS when compared with B27 prevalence of $8 \%$ in Caucasians in general. ${ }^{[}$Other associated diseases are reactive arthritis, psoriatic arthritis, inflammatory bowel disease and acute anterior uveitis. These diseases are so strongly interrelated in the presence of HLA- B27 and called as B27 associated diseases or Seronegative spondyloarthropathies. ${ }^{[3]}$

In present study, HLA-B27/7 antibody was used to study the association between AS and AAU.

\section{Material and Methods}

Inclusion Criteria - The study evaluated hundred patients over a 2 year period at Maharaja Yashwant Rao Holkar hospital Indore. Patients presenting with unilateral or bilateral pain in hip joints, pain in lumbar and sacral region of spine and stiffness along with insidious onset of redness and pain in eye.
Exclusion criteria- The patients having positive rheumatoid factor, age related degenerative changes in bones along with pain and stiffness in small joints like wrist joint were excluded from the study. Those having chronic history of inflammation and pain in eye along with any type of trauma were also not included in the study.

After the clinical diagnosis of AS and AAU, arterial blood samples in EDTA vial were taken for the flow cytometric analysis of HLA-B27/7 antigen.

\section{Results}

Gender Variation - A total of 100 cases with HLA-B27 associated AS and AAU, including 76 males and 24 females were enrolled as shown in table I, giving male and female ratio of 3.1:1, suggesting significantly higher incidence in male population against the female population.

Age Distribution - Patients with age group 06 years and up to 75 years were taken into study. The mean age of onset for male patients was $30.15 \pm 13.0$ years which was significantly younger than female patients with mean age of onset is $42.0 \pm 11.6$ years.

HLA- B27 antigen detection - A total of 100 patients of AS were analyzed for HLA- B27 with the help of flowcytometer in the department of pathology.

Table I shows that 84 patients among were positive for HLA- B27 antigen, including 70 males and 14 females. 
The positive rate of HLA-B27 was 92.0\% (70/76) for males and 58\% (14/24) for females.

Eye involvement - Among 100 patients of AS, 40 were presenting the features of AAU. Among the eyes, most common presentation is unilateral with 28 patients.

Association of AS and AAU - The patient of AS and positive HLA-B27 antigen have higher incidence of Acute anterior uveitis as compared to patients with AS and negative status of HLA- B27 antigen. As shown in table II, 37 (44\%) patients were presented with the clinical features of AAU , AS and Positive HLA- B27 antigen as compared to patients with negative HLA-B27 antigen and AS , only $3(18 \%)$ patients presented with AAU. Therefore risk of developing AAU is 2.4 times higher in patients with HLA-B27 (+) AS as compared to HLA-B27 (-) AS.

Table I : Showing distribution of HLA-B27 Antigen among patients.

\begin{tabular}{|l|c|c|c|c|}
\hline \multicolumn{5}{|c|}{ HLA -B27 antigen Positive Negative } \\
\hline No. of cases & \multicolumn{2}{|c|}{84} & \multicolumn{2}{c|}{16} \\
\hline Sex & M & F & M & F \\
\hline & 70 & 14 & 06 & 10 \\
\hline
\end{tabular}

Table II Details of Acute anterior uveitis in patients of HLA-B27 associated AS

\begin{tabular}{|l|c|c|c|c|}
\hline HLA -B27 antigen & \multicolumn{2}{|c|}{ Positive } & \multicolumn{2}{c|}{ Negative } \\
\hline No. of cases & \multicolumn{2}{|c|}{84} & \multicolumn{2}{c|}{16} \\
\hline AAU & + & - & + & - \\
\hline & 37 & 47 & 03 & 13 \\
\hline
\end{tabular}

\section{Discussion}

In the population of patients with AS approximately $76 \%$ patients are male and rest of the patients are female, giving the ratio of 3.1:1 and the mean age of onset for male patients was $30.15 \pm 13.0$ years which was significantly younger than female patients with mean age of onset is $42.0 \pm 11.6$ years. The incidence of AS when correlated with sex shows the little variation.

Yuqin Wang et al found the ratio of male and female patient is $2.4: 1$ and age of onset is $37 \pm 12$ years. ${ }^{[4]}$

Prevalence of HLA B27/B7 antigen is $84 \%$ in the patients of AS, predominance is seen here also in the male patients. Yang et al found $75.2 \%$ of males with HLA B27/B7 and Wang et al also reported $70.9 \%$ prevalence of HLAB27/B7 in males compared to $29.1 \%$ in females. ${ }^{[4,5]}$
Risk of developing AAU is higher in patients with HLA-B27 (+) AS as compared to HLA-B27 (-) AS. Odd ratio is calculated with the help of SPSS software and it comes out be 3.41 .

HLA- B27 associated AAU mainly affects young population and is more frequently seen in male than in female subjects.

Evaluated data is statistically calculated through SPSS 19.0 software and results are not significant $(\mathrm{p}-0.052$ and $\mathrm{p}<0.05$ is significant) Dominique et al, N. Zebulon et al Li Sun et al and Peizeng Yang et al also discussed the association of HLA-B27, AS and AAU. ${ }^{[6,7,8]}$

\section{Conclusion}

Because uvieitis can be the initial manifestation of Anklyosing spondylitis and especially a key symptom for the diagnosis of AS, the joint work of ophthalmologists and rheumatologists is fundamental to the earlier diagnosis and effective treatment of those patients and to the management of cases.

It's worth emphasizing the need for the accurate rheumatological investigation of HLA-B27 among young males with back pain or stiffness and AAU, it should be periodically repeated for further management and follow up.

\section{Acknowledgement}

I express my feeling of gratitude, respect and appreciation for Prof. Dr. C.V. Kulkarni, Prof and Head of department for his keen supervision, Dr. Ashok Yadav and Dr. Sachin Sharma for guiding me and helping me in technical method, handling of instrument and writing this article.

\section{References}

1. Brewerton DA, Caffrey M, Hart FD, James DCO, Nicholls A, Sturrock RD. Ankylosing spondylitis and HL-A 27. Lancet 1973: 1: 904-7.

2. Brewerton DA, Caffrey M, Hart FD, James DCO, Nicholls A, Sturrock RD. Acute anterio $r$ uveitis and HL-A 27. Lancet 1973:2:994-6.

3. Ebringer A. The cross-tolerance hypothesis, HLA-B27 and ankylosing spondylitis. Br J Rheumatol 1983; 22 :53-66.

4. Wang Y, Xiaoya Lu, Yulin Wang, Liping Mao, Yunfeng Gu, PengfeiChen,Meiqin Zheng, Clinical Analysis Of 240 Pateints With HLA-B27 Associated Acute Anterior Uvieitis. Eye science 2012:27:169-172.

5. Peizeng Yang, Wenjuan Wan, iping Du,Qingyun Zhou, JianQi, Liang Liang, Chaokui Wang, Lili Wu, Aize Kijlstra, clinical features of HLA -B27 positive acute anterior uveitis with or without Ankylosing spondylitis in a Chinese cohort. Br J Opthalmol 2017: 0:1-5. 
6. Monnet D, Breban M, Hudry C, Dougados M, Antoine P. Bre'zin, Ophthalmic Findings and Frequency of extraocular manifestations in patients with HLA-B27 uvieitis. American academy of ophthalmology 2004 :111: 802-809.

7. Zeboulon N, Dougados M, Gossec L, Prevalence and characteristics of uveitis in the spondyloarthropathies. Ann Rheumat Dis 2008:67: 955-959.

8. Li Sun, Rui Wu, Qin Xue, Feng Wang, and Peirong, Risk factors of uveitis in ankylosing spondylitis. Wolters Kluwer Health Medicine 2016 Jul : 95(28)e4233.

*Corresponding author:

Dr. Sweta Tripathi, H.No 16/21 Indra Nagar, P.O- R.K.Purithatipur ,Gwalior (474011) M.P, India

Email: Shweta.angel17@gmail.com

Financial or other Competing Interests: None. 